\title{
Estilo de gestión de los directores y transformación de la praxis pedagógica de
} los docentes

\section{Management style of principals and transformation of teachers' pedagogical praxis}

\author{
Magdiel Azula-Coronel \\ mazulac@unmsm.edu.pe \\ Universidad Nacional Mayor de San Marcos, Lima \\ Perú \\ https://orcid.org/0000-0002-5295-3060
}

Recepción: 24 septiembre 2020

Revisado: 25 de octubre 2020

Aprobación: 16 noviembre 2020

Publicación: 07 de diciembre 2020 
EPISTEME KOINONIA

Revista Electrónica de Ciencias de la Educación, Humanidades, Artes y Bellas Artes
Año III. Vol III. No6. Julio - Diciembre 2020
Hecho el depósito de Ley: FA2018000022
ISSN: 2665-0282
FUNDACIÓN KOINONIA (F.K).
Santa Ana de Coro, Venezuela
Magdiel Azula-Coronel

\title{
RESUMEN
}

El objetivo fue analizar el estilo de gestión de los directores y transformación de la praxis pedagógica de los docentes en instituciones educativas estatales de educación secundaria del Perú. Se circunscribió metodológicamente a un tipo descriptiva con diseño no experimental. El $30 \%$ de la reflexión sobre su práctica por cuanto esto fundamenta la praxis pedagógica. Los estilos de liderazgos influyen en un rol directivo que motive a los docentes a fomentar una praxis pedagógica para la conformación de una educación de calidad en razón de proporcionar un aprendizaje significativo, sin embargo, el docente al no percibirse motivado desde su seguridad salarial y social, tiende a reproducir prácticas pedagógicas que no constituyen un real avance de la calidad de la educación, por ende, del logro de aprendizajes significativos en el estudiante.

Descriptores: Experimento educacional; administración de la educación; planificación de la educación. (Palabras tomadas del Tesauro UNESCO).

\begin{abstract}
The objective was to analyze the management style of the directors and transformation of the pedagogical practice of the teachers in state educational institutions of secondary education in Peru. It was methodologically limited to a descriptive type with a nonexperimental design. $30 \%$ of the reflection on their practice because this bases the pedagogical praxis. Leadership styles influence a directive role that motivates teachers to promote pedagogical praxis for the formation of a quality education in order to provide meaningful learning, however, the teacher does not perceive himself motivated from his salary and social security, tends to reproduce pedagogical practices that do not constitute a real advance in the quality of education, therefore, the achievement of significant learning in the student.
\end{abstract}

Descriptors: Educational experiments; educational administration; educational planning. (Words taken from the UNESCO Thesaurus). 


\section{INTRODUCCIÓN}

En la actualidad el tema de gestión se considera como un aspecto fundamental en el desarrollo de Instituciones Educativas. Son muchos los estudios que demuestran empíricamente la importancia de la dirección en la calidad educativa, definida como el buen funcionamiento de la Institución Educativa, distinguiéndola de otra con características similares con respecto a logros conseguidos, satisfacción y eficacia percibida.

En la dirección, el liderazgo pedagógico es un factor decisivo en un contexto organizativo democrático basado en la participación y la corresponsabilidad. Esto supone un giro en el quehacer directivo superando una función meramente administrativa y/o burocrática. Es decir, que la razón de ser del director debe ser la organización pedagógica y la posibilidad de dinamizar al profesorado y las propuestas curriculares.

Para ello la comprensión de la realidad es un requisito indispensable a la hora de poner en marcha cualquier iniciativa de cambio. En las Instituciones Educativas se muestra cierta resistencia al cambio y a la acción de todo tipo de movimiento innovador. Los cambios en el mundo se concretizan de manera tan rápida y las Instituciones Educativas se quedan estáticas en el tiempo. Cabe en este sentido plantear la siguiente interrogante: ¿Cuáles son los factores que impiden la transformación de las Instituciones Educativas?

Suponemos que hay múltiples factores, pero en esta oportunidad nos limitaremos a determinar el nivel de influencia de la dirección en la real transformación de la praxis pedagógica en las instituciones educativas.

En consecuencia, las personas responsabilizadas con la conducción de una actividad, cualquiera que sea la naturaleza de esta, la función que tenga el que la lleva a cabo, la ejecutan mediante un proceso de dirección. Cada actividad que se lleva a cabo en la Institución Educativa, constituye un componente de todo el sistema que se organiza para dar cumplimiento al fin y a los objetivos/propósitos de la Educación y del nivel; por ello se requiere de un proceso de dirección integrador, que conduzca con eficiencia la acción de todos los factores involucrados en la Institución. 


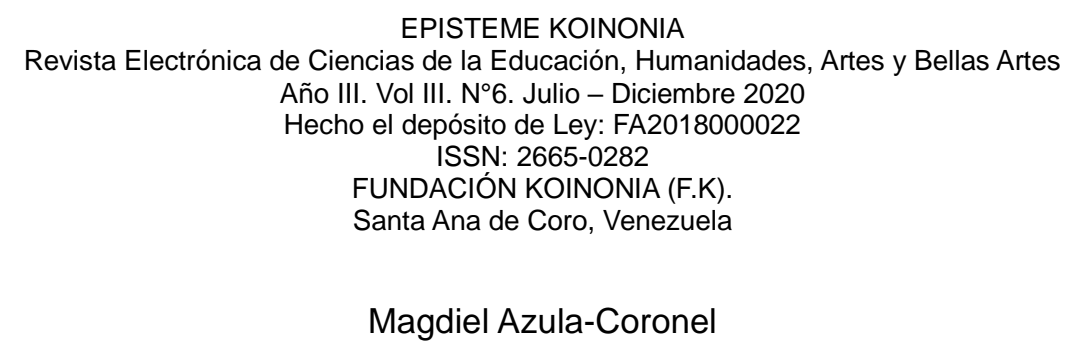

Esta misión necesariamente tiene que asumirla el director de la Institución Educativa, en este sentido buscamos investigar si realmente los directores de las Instituciones Educativas estatales de Educación secundaria, orientan a las Instituciones Educativas sobre bases sólidas de dirección y se concreta en una verdadera transformación de la praxis pedagógica en sus aulas y el contexto de la comunidad educativa.

La importancia de este estudio radica en que, al conocerse los resultados, se planifique capacitaciones permanentes, prácticas y vivenciales sobre acciones concertadas y liderazgo a los directores de Instituciones Educativas, se considere en la designación de los directores de las Instituciones Educativas el perfil personal y profesional que requiere el cargo de director y de esta manera garantizar la transformación de las Instituciones Educativas.

En este sentido, se tiene por objetivo analizar el estilo de gestión de los directores y transformación de la praxis pedagógica de los docentes en instituciones educativas estatales de educación secundaria del Perú.

\section{MÉTODO}

La investigación se circunscribió metodológicamente a un tipo descriptiva con diseño no experimental, observándose y describiéndose las variables de estudio, tal como se desarrollaron en la realidad, no existiendo manipulación deliberada por parte del investigador. Se trabajó con una muestra poblacional de 300 personas distribuidas entre directivos y docentes de de 08 instituciones educativas públicas de educación secundaria, que cuentan don director designado, del distrito de Bambamarca, provincia de Hualgayoc, departamento de Cajamarca del Perú.

Se empleó la técnica de la encuesta para la recopilación de información, a través de un cuestionario en escala de Likert con 40 ítems y cinco alternativas de repuestas, dirigido tanto a directivos como docentes, de ese modo, se validó a través del juicio de tres expertos para efectos de contenido, luego se realizó prueba piloto con la finalidad de calcular la fiabilidad en razón del coeficiente Alfa de Cronbach, obteniéndose un resultado 
de 0,86 siendo considerable confiable para su aplicación. La información recopilada, se procesó mediante estadística descriptiva, presentándose tabla de resultado porcentaje y frecuencia en razón de cada variable de investigación.

\section{RESULTADOS}

En razón de la recopilación y procesamiento de la información, se presentan los siguientes resultados:

\section{Tabla 1}

Estilos de gestión del director.

\begin{tabular}{|c|c|c|}
\hline & $\mathrm{fr}$ & $\%$ \\
\hline Estilo autocrático del director & 102 & 34 \\
\hline Estilo democrático del director & 101 & 34 \\
\hline Estilo liberal o laissez faire del director & 97 & 32 \\
\hline & 300 & 100 \\
\hline
\end{tabular}

Los estilos democrático y autocrático en un 34\% mientras que el estilo liberal en $32 \%$ visualiza que existe una práctica heterogénea, lo cual indica que los directores desarrollan diversos estilos de gestión, de ese modo, cada uno influye en la transformación pedagógica en razón del direccionamiento adscrito al modo de liderazgo direccionado por el director.

\section{Tabla 2.}

Praxis pedagógica del docente.

\begin{tabular}{|c|c|c|}
\hline & $\mathrm{fr}$ & $\%$ \\
\hline Conocimiento y comprensión & 67 & 22 \\
\hline Planificación de la enseñanza & 85 & 28 \\
\hline Creación de un clima propicio para el aprendizaje & 59 & 20 \\
\hline Reflexión sobre su práctica & 89 & 30 \\
\hline Total & 300 & 100 \\
\hline
\end{tabular}




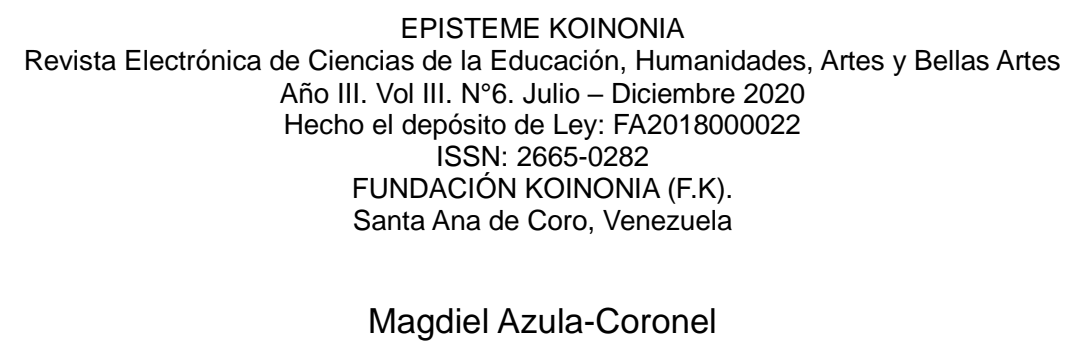

Se destaca en los resultados el $30 \%$ de la reflexión sobre su práctica por cuanto esto fundamenta la praxis pedagógica, complementada en $28 \%$ desde una planificación que contribuye a una enseñanza transformadora.

\section{DISCUSIÓN}

Los estilos de liderazgos en sus diversas acepciones, tienen a estimular el comportamiento de los miembros de la organización, aunque cada uno persigue el fin de ser productivos, cumplir con las metas asignadas, lo que cambia en esencia, es el proceso de cómo se adquieren esas proyecciones en razón del estilo aplicado, en cierto modo, se puede indicar como un estímulo - respuesta, por consiguiente es de suma importancia conocer el desarrollo de los estilos de liderazgos para conocer la gestión organizacional (Escandon-Barbosa \& Hurtado-Ayala, 2016).

Es así que el liderazgo directivo escolar, influye en los docentes para que puedan articular los planes institucionales en favor de cumplir las metas organizacionales (Lugo \& Villasmil Ferrer, 2019). De ese modo, los directores tienen la oportunidad de gestionar en favor de una educación de calidad basada desde la construcción de saberes de los sujetos integrantes de la escuela, por cuanto cada uno asume el rol correspondiente en razón de favorecer el aprendizaje de los estudiantes, para lo cual, podría comprenderse la calidad en razón de tres niveles:

En primer nivel denominado esencial, que sirve de pilar para la construcción subjetiva en torno al tema, e incluye fundamentalmente el compromiso y la preocupación personal por los estudiantes. El segundo nivel, denominado formativo, incluye la entrega de valores y de habilidades para la vida. El tercer nivel, por su parte, denominado instrumental, incluye los logros de aprendizaje y los componentes de la gestión escolar (Torche, et al., 2015, p. 131).

Se destaca la construcción del principio de calidad educativa entre directivos y docentes, como una conjugación intersubjetiva de comprensión de la realidad académica en virtud de propiciar un aprendizaje significativo a los estudiantes, prosiguiéndose con una entrega valorativa de concepciones éticas para la conformación de un imaginario social 


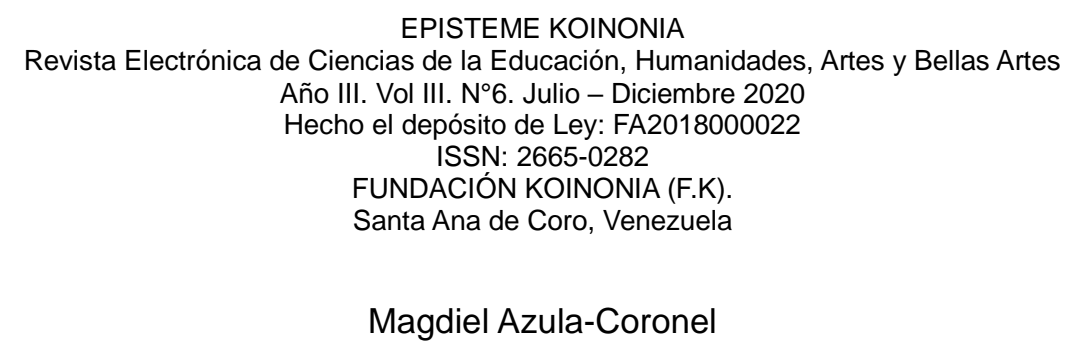

constituido desde la praxis pedagógica como eslabón para la generación de un ciudadano de bien, retomándose así, el rol socializador de la escuela, cerrándose con el logro de los procesos cognitivos - procedimentales propuestos en la planificación.

En este sentido, el liderazgo directivo en primer lugar, no debe estar enfocado en la conducción unidireccional de los objetivos pedagógicos propuestos en el currículo, sino, en la de formación de un ser integral, para lo cual, (Murillo \& Martínez-Garrido, 2018), consideran importante fomentar el aspecto socio - afectivo del estudiante, potenciándose su auto concepto como eje fundamental para el crecimiento personal, situación que debe darse sí el directivo - docente, ha asumido esta postura emocional en sus vidas, las han intercambiado como pares profesionales, para luego en conjunto ser reproducida a los estudiantes, de no ser así, se desarrollaran aisladamente, pero no en bloque uniforme, ante lo cual, la organización educativa vería mermado su gestión directiva.

Para lograr lo planteado, no siempre existen las condiciones, existen factores externos a la organización educativa que inciden en la gestión de un liderazgo enfocado en estilos tradicionales como autocrático, democrático, laissez faire (Sánchez-Reyes \& BarrazaBarraza, 2015), lo cual impide la generación de liderazgos emergentes o de menor clasismo, siendo considerable el estudio particular en las organizaciones sobre los elementos indiciadores en la negación de un líder transformador.

Una opción es el liderazgo distribuido planteado por (Maureira, et al., 2014), el cual procura romper la verticalidad directiva para involucrar la horizontalidad, así cada docente tiene la co responsabilidad de asumir funciones directivas, conformándose a su vez un equipo de alto nivel organizacional, lo cual no resulta fácil, por cuanto se requiere del trabajo colaborativo de todos (Herrera-Huiracocha \& Salinas, 2018), la cohesión organizacional contribuye a formular el intercambio de habilidades, destrezas, competencias, entre los directivos - docentes, con la finalidad de propiciar un clima organizacional acorde para la consecución de metas comunes. 


\section{Magdiel Azula-Coronel}

El clima organizacional óptimo entre directivos y docentes, tiende a favorecer las relaciones interpersonales y así, proyectar un mejor desenvolvimiento para desempeñar el proceso de enseñanza - aprendizaje, siendo este un reflejo del liderazgo ejercido, articulándose un desenvolvimiento donde cada actor, trabaja en el logro de las metas comunes en virtud de conseguir una educación de calidad (Maya, et al., 2019), en complemento, (Sierra-Villamil, 2016), propone ejercer el liderazgo educativo desde una perspectiva sostenible, esta proyecta trabajar un currículo acorde a las exigencias de la agenda 2030 donde se requiere una educación cónsona con la sociedad global, basada desde el enfoque de sostenibilidad.

Un liderazgo sostenible, involucra la conexión entre diversas competencias multidisciplinarias para configurar una acción educativa basada en un aprendizaje a lo largo de la vida (Da-Trindade-Prestes \& Santos-Diniz, 2015), es así que el directivo, tiene en esencia, la responsabilidad de promover en el docente, la reflexión para asumir una praxis pedagógica en conformidad a los tiempos presentes - venideros, donde los cambios sociales van a influir directamente en la modalidad de estudio, es así que se necesitará la generación de un currículo que brinde respuestas a tales exigencias, sin embargo, este debe emerger desde la propia vivencia de los docentes.

Es decir, se requiere diseñar un currículo apegado a las vivencias contextuales del docente en relación al estudiante y su entorno social, cada escuela desde esta perspectiva, se constituye en una micro sociedad con su propia dinámica que abordar, posiblemente similar a otras, pero en el fondo, realmente distantes, es allí donde la reflexión del docente, permite constituir elementos para una propuesta educativa flexible, en relación a una educación sostenible de calidad.

Sin embargo, esta apuesta no es novedosa, pero ha resultado infructuosa lograr, por cuanto el docente al no percibirse motivado desde su seguridad salarial y social, tiende a reproducir prácticas que no constituyen un real avance de la calidad de la educación, por ende, del logro de aprendizajes significativos en el estudiante (Castro, et al., 2009), es en este punto donde factores externos a la institución educativa, afectan al rendimiento 


\section{Magdiel Azula-Coronel}

integral de la misma, así como a la concepción de nuevas vertientes curriculares, lo que denota no solo la necesidad de contar con estilos de liderazgo directivo acorde a propiciar un excelente ambiente de trabajo, sino, de políticas educativas concertadas en elevar la calidad de vida del docente, solo así, podrá existir un giro para el cumplimiento de las labores pedagógicas en razón de articular un proceso de aprendizaje acorde a las múltiples necesidades globales que envuelven a la sociedad de hoy.

\section{CONCLUSIÓN}

Los estilos de liderazgos influyen en un rol directivo que motive a los docentes a fomentar una praxis pedagógica para la conformación de una educación de calidad en razón de proporcionar un aprendizaje significativo. Sin embargo, no solo basta trabajar en bases a los estilos clásicos de liderazgo, es necesario probar nuevos enfoques en razón de los avances organizacionales adaptados a la gerencia educativa, así como existen factores externos a las instituciones, entre los cuales, se encuentra la seguridad social del docente, siendo este un factor primordial por cuanto al no existir un salario digno, se proyecta la repetición de una práctica pedagógica monótona que no proyecta la transcendencia hacia nuevos modelos o estilos pedagógicos, viéndose truncada, la efectividad hacia una educación de calidad y contextualizadas a las necesidades sociales.

\section{FINANCIAMIENTO}

No monetario

\section{AGRADECIMIENTOS}

A la Universidad Nacional Mayor de San Marcos; por el apoyo en la realización de esta investigación. 
EPISTEME KOINONIA

Revista Electrónica de Ciencias de la Educación, Humanidades, Artes y Bellas Artes

Año III. Vol III. N6. Julio - Diciembre 2020

Hecho el depósito de Ley: FA2018000022

ISSN: 2665-0282

FUNDACIÓN KOINONIA (F.K).

Santa Ana de Coro, Venezuela

Magdiel Azula-Coronel

\section{REFERENCIAS}

Escandon-Barbosa, D, \& Hurtado-Ayala, A. (2016). Influencia de los estilos de liderazgo en el desempeño de las empresas exportadoras colombianas [Influence of leadership styles in the performance of Colombian export companies]. Estudios Gerenciales, 32(139), 137-145.

Lugo, N., \& Villasmil Ferrer, J. (2019). Liderazgo directivo como factor de mejoramiento en la calidad educativa [Executive leadership as a factor for improving educational quality]. EPISTEME KOINONIA, 2(4), 4-29. http://dx.doi.org/10.35381/e.k.v2i4.521

Torche, P, Martínez, J, Madrid, J, \& Araya, J. (2015). ¿Qué es "educación de calidad" para directores y docentes? [How is "quality education" defined by principals and teachers?]. Calidad en la educación, (43), 103135. https://dx.doi.org/10.4067/S0718-45652015000200004

Murillo, F. J, \& Martínez-Garrido, C. (2018). Factores de aula asociados al desarrollo integral de los estudiantes: Un estudio observacional [Teaching factors associated with the comprehensive development of students: An observational study]. Estudios pedagógicos (Valdivia), 44(1), 181205. https://dx.doi.org/10.4067/S0718-07052018000100181

Sánchez-Reyes, B, \& Barraza-Barraza, L. (2015). Percepciones sobre liderazgo [Perceptions of leadership]. Ra Ximhai, 11(4),161-170.

Maureira, O, Moforte, C, \& González, G. (2014). Más liderazgo distribuido y menos liderazgo directivo: Nuevas perspectivas para caracterizar procesos de influencia en los centros escolares [More distributed leadership and less directive leadership. New perspectives to characterize processes of influence in educational centers]. Perfiles educativos, 36(146), 134-153.

Herrera-Huiracocha, C, \& Salinas, E. (2018). Equipos de alto desempeño para pequeñas y medianas empresas [High performance teams for small and medium enterprises]. Revista Universidad y Sociedad, 10(1), 300-304.

Maya, E., Aldana Zavala, J., \& Isea Argüelles, J. (2019). Liderazgo Directivo y Educación de Calidad [Executive Leadership and Quality Education]. CIENCIAMATRIA, 5(9), 114 - 129. https://doi.org/10.35381/cm.v5i9.102 
EPISTEME KOINONIA

\author{
Revista Electrónica de Ciencias de la Educación, Humanidades, Artes y Bellas Artes \\ Año III. Vol III. №6. Julio - Diciembre 2020 \\ Hecho el depósito de Ley: FA2018000022 \\ ISSN: 2665-0282 \\ FUNDACIÓN KOINONIA (F.K). \\ Santa Ana de Coro, Venezuela \\ Magdiel Azula-Coronel
}

Sierra-Villamil, G. M. (2016). Liderazgo educativo en el siglo XXI, desde la perspectiva del emprendimiento sostenible [Educational leadership in the 21st century, from the perspective of sustainable entrepreneurship]. Revista Escuela de Administración de Negocios, 81, p. 111. doi:10.21158/01208160.n81.2016.1562

Da-Trindade-Prestes, E, \& Santos-Diniz, A. (2015). Educación y aprendizaje a lo largo de la vida: los adultos y la enseñanza superior [Education and lifelong learning: adults and higher education]. Sinéctica, (45), 1-20.

Castro, E, Rosario, P, \& Morillo, R. (2009). La praxis educativa: Una aproximación a la realidad en el aula [Educational praxis: An approach to reality in the classroom]. Revista Venezolana de Gerencia, 14(45), 125-143.

(C2020 por el autor. Este artículo es de acceso abierto y distribuido según los términos y condiciones de la licencia Creative Commons Atribución-NoComercial-Compartirlgual 4.0 Internacional (CC BY-NC-SA 4.0) (https://creativecommons.org/licenses/by-nc-sa/4.0/). 